\title{
EL DESARROLLO DE LA ÉTICA EMPRESARIAL ANTE LOS AVANCES DE LA NEUROCIENCIA ORGANIZACIONAL Y LA NEUROÉTICA
}

\author{
ELSA GONZÁLEZ ESTEBAN \\ Universitat Jaume I
}

\begin{abstract}
RESUMEN: La neurociencia organizacional y la neuroética están logrando un gran avance en el descubrimiento tanto de la cognición como del comportamiento humano que invitan a repensar algunos de los presupuestos clásicos sobre los que se han desarrollado las éticas aplicadas. Este estudio se centra en analizar cómo afectan los avances neurourocientíficos a nuestra comprensión de la ética empresarial y, en especial, en su nivel organizativo. Para alcanzar este objetivo se estructura el trabajo en dos pasos. Por una parte, el estudio examina si tales avances minan o socavan los cimientos normativos de la ética empresarial. Y, por otra parte, en un segundo paso se intentan descubrir las implicaciones que tales avances tienen para la ética empresarial en su desarrollo práctico.
\end{abstract}

PALABRAS CLAVE: ética empresarial, racionalidad, emoción, neurociencia organizacional, neuroética.

\section{The development of Business Ethics in front of the progress of organizational neuroscience and neuroethics}

ABSTRACT: Organizational neuroscience and neuroethics are proving to have already made a number of advances in the discovery of both cognition and human behaviour. Those progresses invite us to rethink some of the classical assumptions on which have been developed applied ethics. This article focuses on the analysis about how the progresses of the neurosciences affect our understanding of business ethics and its future development, specially in its organizational level. In order to achieve this objective, the paper is structured in two steps. On the one hand, it considers whether such developments undermine or erode normative foundations of business ethics. On the other hand, it tries to discover the implications of such developments have for business ethics in its practical development.

KEY WORDS: business ethics, rationality, emotion, organizational neuroscience, neuroethics.

\section{1. Ética Empresarial y Neurociencia Organizacional ${ }^{1}$}

La ética empresarial es una disciplina que nace en los años 70 del siglo xx con el objetivo de orientar a las empresas a gestionar su dimensión ética. Es decir, a reconocer y gestionar los recursos morales —expectativas relacionalesde los que dispone la organización y que es necesario hacerlo conscientemente para tener éxito en el medio y largo plazo generando valor para todos sus stakeholders o grupos de interés que lo configuran (Freeman, 1984; García-Marzá, 2004; González Esteban, 2007 y Freeman et al. 2010). En definitiva la ética

1 Este estudio se inserta en el Proyecto de Investigación Científica y Desarrollo Tecnológico FFI2013-47136-C2-2-P, Política, empresa y educación desde la Neuroética Aplicada , financiado por el Ministerio de Economía y Competitividad, y en las actividades del grupo de investigación de excelencia PROMETEO/2009/085 de la Generalitat Valenciana. 
empresarial desde sus inicios ha tratado de elaborar tanto una fundamentación normativa como caminos para su desarrollo práctico para que las organizaciones tomen decisiones prudentes, justas y responsables generando una nueva cultura empresarial (Cortina et al., 1994; Cortina, 2012).

En la actualidad, como se muestra en el presente trabajo, algunos de los supuestos clásicos sobre los que se sustenta la ética empresarial están siendo cuestionados desde el avance de las neurociencias. Para dilucidar qué aspectos deberían ser considerados de tales avances por parte de la ética empresarial, en su nivel organizativo, se darán seis pasos en este estudio. En primer lugar, se realizará una aproximación a las pretensiones y avances de la neurociencia organizacional desde sus primeros pasos en el año 2007; en segundo lugar, se cuestionará si tales avances socavan los cimientos de fundamentación de la ética empresarial o simplemente lo que hacen es ampliar y mejorar la comprensión de las bases fisiológicas de las actitudes y del comportamiento humano en el nivel de las relaciones organizativas; en tercer lugar, se muestran los modelos de toma de decisiones éticas que han incorporado ya algunos de los principales avances neurocientíficos y se apunta que si bien son relevantes no implican abandonar los modelos de racionalidad crítica; en cuarto lugar, se abordan las cuatro estrategias que se están desarrollando para incorporar las emociones en las concepciones racionalistas de las organizaciones; en quinto lugar, se identifican seis ámbitos de la ética empresarial organizativa que deberían ser reconsiderados a la luz de los avances de la neuroética y la neurociencia organizacional; y, en sexto y último lugar se concluye que si bien tales aportaciones es necesario considerarlas se deben tomar algunas de sus aportaciones con cautela. Comencemos viendo porqué adentrándonos en la neurociencia organizacional.

La neurociencia organizacional se propone por primera vez en 2007 por Michael Butler y Carl Senior como un nuevo campo de investigación capaz de generar nuevo conocimiento que transcienda los discursos separados de la neurociencia cognitiva y de la teoría organizacional. Según estos autores el gran debate que se esconde tras la neurociencia organizacional es el papel que juegan de un lado la naturaleza y de otro la cultura y la educación tanto en los individuos como en las organizaciones. La neurociencia cognitiva organizacional se puede definir como la utilización de instrumentos como la neuroimagen para analizar el cerebro humano y comprender tanto su funcionamiento cerebral como su comportamiento humano dentro de la aplicación a un marco organizativo (Butler y Senior, 2007: 8). Sus avances se muestran por distintos autores como relevantes en tres grandes ámbitos: en el conocimiento de la estructura de los marcos sociales con el descubrimiento de los sistemas de neuronas espejo; en la influencia de las procesos no conscientes en nuestras actitudes y comportamientos; y, en la resolución de debates conceptuales (Becker y Cropanzano, 2010; Becker et al., 2011; Cropanzano y Becker, 2013). A continuación, se da cuenta brevemente de estos tres ámbitos.

En primer lugar, uno de los supuestos sobre los que se desarrolla la neurociencia organizacional afirma que tras muchos de los comportamientos 
adaptativos de grupo se encuentra el funcionamiento de las neuronas espejo. La interpretación de las mismas que se realiza es que nuestros cerebros están conectados para hacernos abiertos a las influencias sociales por razones adaptativas y la existencia de las neuronas espejos es la base fisiológica que explica dicho comportamiento (Becker y Cropanzano, 2010: 1057; Becker et al., 2011: 838-940). Por tanto, tenemos una base fisiológica que nos predispone a un aprendizaje vicario que cabe explotar en el nivel organizativo. Este conocimiento permite que se conozca mejor cómo interactúan las personas en la generación de climas organizativos y la relevancia que tienen para la creación de equipos de trabajo. Cuando un grupo interactúa aprende de las valoraciones y comportamientos de los demás miembros del grupo. Esto podría dar lugar en ocasiones a actitudes o comportamientos que son adaptativos para el grupo, pero no necesariamente para el desarrollo del bien interno de la organización, como es el caso de la corrupción, el nepotismo o las malas prácticas. En este aprendizaje vicario los afectos - sentimientos y emociones- juegan un papel muy relevante dado que permiten que se produzcan aprendizajes inconscientes entre los miembros del grupo de modo muy rápido.

En segundo lugar, los avances neurocientíficos y neuropsicológicos también han sugerido que mucho de lo que la gente aprende durante el proceso de socialización es procesado no-conscientemente por nuestro cerebro. De modo que en muchas ocasiones las personas no somos conscientes de las actitudes implícitas que están tras nuestros comportamientos. Estas actitudes implícitas acurren automáticamente y muy rápidamente dentro de nuestro cerebro y sólo cuando entramos en un momento de confusión o de confrontación entramos en una racionalización de las mismas (Haidt, 2012).

La neurociencia afirma que existe una explicación neural con base fisiológica que da cuenta de la diferencia existente entre las actitudes explícitas y las actitudes implícitas. En cuanto a las explícitas los estudios apuntan a un razonamiento deliberativo que surgen en las estructuras del control del lóbulo frontal ejecutivo que son bastante nuevas en términos evolutivos, mientras que las actitudes implícitas están producidas en regiones anteriores filogenéticamente. En el ámbito organizacional esto podría explicar porqué los cambios organizativos son tan difíciles de acometer: porque las empresas y sus directivos se centran sobre las actitudes explícitas y fallan en valorar y trabajar sobre las actitudes implícitas. En ocasiones ni los mismos trabajadores son conscientes de sus reticencias al cambio, puesto que las actitudes implícitas surgen primero y son capaces de entorpecer el surgimiento de otras actitudes implícitas o de procesos conscientes. También podría explicar en entornos multiculturales la discriminación en el trabajo en forma de intolerancia así como la disociación que se produce entre actitudes implícitas y explícitas en este terreno que requieren de una gestión consciente y deliberada desde el nivel de políticas de la organización.

En último lugar, como ya se ha mencionado, cabe señalar que la neurociencia organizacional se plantea como un nuevo conocimiento que puede mejorar nuestra comprensión de múltiples aspectos en las relaciones empresariales y 
de las motivaciones para actuar que subyacen a las valoraciones en las organizaciones. Así por ejemplo Folger y Salvador (2008: 1128) afirman que en la literatura organizacional existen dos grandes posiciones respecto a la justicia en los entornos laborales. De un lado están los autores que mantienen que los individuos a menudo valoran la justicia por su propio bien es decir que independiente del resultado que produzca en ellos o en la organización es considerada un valor en sí mismo como finalidad. Mientras que, por otro lado, hay autores que mantienen que la justicia no es un valor por sí mismo sino un medio para que el individuo adquiera ventaja. Por lo que sólo es el propio interés del individuo el que mueve a defender la justicia. Sin duda alguna el debate sobre el propio interés es complejo e implica a numerosas disciplinas que incluyen en este ámbito a la filosofía, la psicología y la dirección y administración de empresas. Sin embargo, lo que apunta el avance neurocientífico y los experimentos neurocientíficos es que estamos preparados para cooperar y que tenemos un sentido de la justicia innato que precede a la racionalización cognitivista posterior. En este sentido, se establece la necesidad de revisar esa ingenua visión del agente racional económico como maximizador de su propia utilidad, lo cual deja el egoísmo como única guía posible de sus comportamientos y decisiones (Calvo y González Esteban, 2012: 113; Conill, 2012: 60). Pero sobre todo lo que resulta interesante, a mi modo de ver, para la revisión de los presupuestos trabajados en la Ética Empresarial como disciplina es que los estudios neurocientificos señalan que tenemos una sensibilidad moral hacia la justicia que está relacionada con las propias respuestas emocionales.

\section{2. ¿ES LA NEUROCIENCIA ORGANIZACIONAL UNA REVOLUCIÓN PARA LOS CIMIENTOS DE LAS ORGANIZACIONES?}

Si bien es cierto que la neurociencia organizacional ha surgido con gran fuerza y está evolucionando rápidamente, en el fondo, como sugieren Lindebaum y Zundel (2013), no estamos frente a una revolución en el conocimiento organizacional. Estos autores critican la propuesta de la neurociencia organizacional tal y como se ha desarrollado hasta el momento especialmente por autores como Cropanzano y Becker. Consideran que sus avances se han llevado a cabo desde posiciones reduccionistas dado que han eliminado en sus planteamientos normativos y desarrollos prácticos los aspectos relacionales y recursivos existentes en las organizaciones. De modo que se podría hablar de un empobreciendo más que de una ampliación de los estudios organizacionales.

Y ¿cómo se produce este empobrecimiento? Sobre todo a través de tres modos de aproximarse a la neurociencia organizacional. La primera consiste en reducir el comportamiento organizacional a las bases biológicas y neurales del cerebro sin tener en consideración el entorno o la cultura. En segundo lugar, trabajando sobre experimentaciones que son binarias en la respuesta ofrecida en el estudio, cuando la realidad es múltiple en cursos de acción. En tercer 
lugar, por considerar que existe un acceso privilegiado a la intención de las personas como si la conciencia en el cerebro se pudiera «leer», cuando no es así dado que hay problemas de interpretación de los datos que ofrecen las lecturas neurocientíficas (Lindebaum y Zundel, 2013)².

Y aunque algunos de los precursores de la neurociencia organizacional reconocen que existen peligros suscribirían las palabras de Cropanzano y Becker cuando afirman que:

«(...) el mayor peligro ético que está presionando a la neurociencia no son los métodos en sí mismo sino en la sobreestimación de las personas o de la promoción excesiva del poder de estos métodos. Mientras que los investigadores no son inmunes a este peligro, la prensa de masas y el público en general han sido susceptibles particularmente del aura de la neurociencia» (Cropanzano y Becker, 2013: 307).

Como se aprecia en estas palabras no se considera que exista un necesidad de revisar las técnicas, experimentos o métodos utilizados por la neurociencia sino las interpretaciones que de ellos se hacen especialmente por quienes no acaban de entender correctamente las implicaciones que sus avances producen (Salles, 2013; Pallarés Dominguez, 2013). Por tanto, sí que se reconoce que hay que andar con cautela, pues es cierto que en ocasiones se realizan interpretaciones apresuradas que pueden llevar a confundir lo que es la explicación del comportamiento organizativo con su justificación u orientación normativa basada en una explicación naturalista o fisiológica.

Por lo que a pesar de las limitaciones y críticas recibidas el avance de la neurociencia organizacional se sigue desarrollando y presentando como un avance que tendrá repercusión en la mejora de la vida de las personas (Cropanzano y Becker, 2013: 307) aunque reconocen que tiene limitaciones. Pero su argumento se sustenta en la promesa de una mejor comprensión de los fenómenos organizativos, como el liderazgo, la toma de decisiones, la cultura o clima organizacional, etcétera, que permita una gestión más efectiva teniendo en cuenta la base neural ofrecida por la neurociencia. Así como también en la posible detección de problemas antes de que estos se produzcan en base a la utilización de las técnicas neurocientíficas, por ejemplo, en la selección de personal, que permitan tomar decisiones sobre si contratar a personas con tengan intuiciones morales xenófobas o racistas. Siendo relevantes ambas cuestiones para la ética empresarial organizativa es en la primera en la que centra la atención este estudio, puesto que la segunda implicaría también profundizar en la capacidad que tienen estos métodos neurocientíficos para adentrarse en la conciencia de las personas así como sobre las interpretaciones que son necesarias llevar a cabo sobre los datos recogidos, entre otras cuestiones (Evers, 2009 y Cortina, 2012b).

2 Para profundizar en las críticas a la perspectiva neurocientificista planteada como método de justificación o normatividad de las ciencias sociales ver Evers, 2009; CortinA, 2011; Salles, 2013 y Pallarés Dominguez, 2013. 
Los temas centrales que han emergido con su avance en el conocimiento neurocientífico del cerebro vinculados a la ética empresarial se pueden centrar especialmente en: las formas de cognición en las tomas de decisiones éticas en la empresa; la dimensión inconsciente en los procesos de toma de decisiones; el papel de la emoción en la ética empresarial; la configuración del liderazgo ético; la revisión de los procesos formativos en gestión y dirección de empresas; y, la generación de estructuras que desincentiven la corrupción en las empresas (Salvador; Folger, 2009: 4 y Weaver et al. 2014: 102).

La ética empresarial, como se apuntaba al inicio de este artículo, tiene la tarea de justificar porqué hay que actuar en un sentido de prudencia, justicia y responsabilidad en las relaciones con los stakeholders y promover caminos prácticos que faciliten una gestión de la ética en las organizaciones. Se puede afirmar que uno de los supuestos básicos que subyace a todas las propuestas de ética empresarial es que el ser humano es capaz de pensar y actuar racionalmente en esa tríada: prudencia, justicia y responsabilidad. De modo que una de las tareas en las que se ha dedicado mucho empeño en estas cuatro décadas es en dotar tanto de fundamentación normativa como de propuestas metodológicas para que la organización alcanzara a través de su diseño organizativo, sus estrategias, su cultura y su política una nueva cultura empresarial de carácter ético (Cortina, et al., 1994; García-Marzá, 2004; González, 2007). Por tanto, dentro de los objetivos programáticos de la ética empresarial, la neurociencia organizacional no es una revolución del conocimiento de las organizaciones aunque sí que invita a replantear algunos de los núcleos gordianos de la ética empresarial tal y como se han planteado desde sus inicios, centrada en las tomas de decisiones cognitivas racionalistas y la generación de relaciones centradas sobre todo en los intereses racionales.

3. REPENSANdo los MOdelos de tOMA DE DECISIONES Y DE COMPORTAMIENTO ÉTICO EN LAS ORGANIZACIONES A LA LUZ DE LA NEUROÉTICA DE LA CIENCIA ${ }^{3}$

Los modelos de procesos de toma de decisiones han estado marcados por el marco cognitivista de los mismos tanto si en las decisiones hay un carácter ético en juego como si no. En el año 1991 Thomas Jones realizó un estudio para determinar cuál era el modelo de toma de decisiones que subyacía a las propuestas de ética empresarial y determinó que este era el propuesto por

3 Se sigue en este punto a Adela Cortina quien entiende que la neuroética de la ciencia es aquella que se ocupa de las bases neuronales de la agencia moral y que refiere al impacto del conocimiento neurocientífico en nuestra comprensión de la ética misma. Por lo que así entendida la neuroética trataría sobre las bases cerebrales de la conducta moral y se preguntaría por los fundamentos filosóficos de la obligación moral (Cortina, 2011:46). A diferencia de la ética de la neurociencia que se preocupa de desarrollar un marco ético para regular la conducta en la investigación neurocientífica y en la aplicación de los conocimiento neurocientíficos a los seres humanos. (Cortina, 2011:39-47). 
James Rest por primera vez en 1979 y desarrollado posteriormente en 1986. Este modelo se sustenta en la teoría cognitivista de Lawrence Kohlberg y se corresponde con un marco deliberativo racional que posee un apoyo empírico considerable. Este modelo incluye cuatro fases: (1) el reconocimiento e interpretación de que una situación presenta una elección moral; (2) elaboración de un juicio moral que surge de la deliberación sobre la situación; (3) formulación de una intención de actuar de acuerdo con tal juicio moral; y, (4) un comportamiento y compromiso moral.

Sin embargo, los avances de la neuroética (Damasio, 1996, 2001 y 2010) así como de la biología evolutiva (Flack y de Waal, 2004) ponen en cuestión, como se verá a continuación, este modelo cognitivista cuando de toma de decisiones éticas se habla. Lo que hay de nuevo con los avances de la neurociencia de la ética es descubrir que en los procesos de toma de decisiones de naturaleza ética determinadas áreas cerebrales entran en acción mediante conexiones neurales para dar lugar a todo el proceso de toma de decisión ética que conduce finalmente a la acción. Las estructuras que se incluyen entre las de mayor activación en los experimentos cuando a los participantes se les hace interactuar ante estímulos en los que las cuestiones morales están en juego se encuentran: el lóbulo frontal —que incluye el complejo orbitofrontal medial y el córtex prefrontral dorsolateral- las estructuras límbicas subcorticales —que incluyen la amígdala y el hipocampo- así como otras estructuras cerebrales por ejemplo, la región del surco temporal superior posterior-. Además todas estas áreas parece ser que son también las que son cruciales para la percepción social, el comportamiento y el cumplimiento respecto a las normas (Salvador y Folger, 2009:6).

De este modo una de las cuestiones centrales que ha ayudado a comprender mejor el avance de la neuroética de la ciencia es que en los procesos de toma de decisiones actúan diferentes partes del cerebro al mismo tiempo que tienen que ver tanto con la razón-deliberación como con la emoción-intuición (Lozano Aguilar, 2013). Y, los estudios apuntan a la emoción como mediadora y moderadora de los procesos organizacionales (Weaver et al., 2014).

Así pues, la emoción ha recibido al menos desde la década de los 90 una gran atención y parece que desde el ámbito neurocientífico se haya llegado a un cierto consenso sobre que la emoción implica una interacción de sistemas neurales cognitivos y no-cognitivos. Por tanto que la emoción debe verse como una integración de subsistemas adaptativos e innatos derivados de las necesidades evolutivas para la supervivencia (LeDoux, 1999; Damasio, 1999; Morgado 2012). En esta línea Damasio (1999) diferencia entre las emociones primarias, que implican emociones necesarias para la supervivencia y basadas en el sistema límbico del cerebro y emociones secundarias, donde el neocortex procesa información de alto nivel. Estos avances se han visto reflejados en la literatura de la ética empresarial de la toma de decisiones bajo dos grandes modelos: el modelo afectivo-cognitivo y el modelo neurocognitivo. A continuación se da cuenta de ellos. 


\subsection{Modelo afectivo- cognitivo de Gaudine y Thorne}

Teniendo en cuenta estos avances en el año 2001 Gaudine y Thorne ofrecen un modelo afectivo-cognitivo del proceso de toma de decisiones en el lugar de trabajo ${ }^{4}$. Este modelo contempla dos dimensiones de la emoción que actúan en el proceso de toma de decisiones, por un lado, el «estímulo» —arousal- y, por otro, el «sentimiento»-«felling state». El estudio empírico que desarrollan basado en este modelo muestra que estas dos dimensiones de la emoción influyen en la propensión individual a identificar los diferentes elementos o etapas de los procesos de toma de decisiones éticas tal y como Rest (1979 y 1986) los identifica. De modo que se comprueba que la experiencia del estímulo y sentimiento positivo permite a los individuos resolver dilemas éticos de una manera consistente con unas estructuras morales cognitivas sofisticadas. Es decir, sobre todo se centra en cómo la emoción influye en la elaboración del juicio moral, tercera parte del proceso de toma de decisiones propuesto por Rest. Desde este modelo y su comprobación empírica se afirma que si bien el proceso de toma de decisiones ética en las organizaciones es cognitivo, dicha cognición está influida siempre por aspectos emotivos que incidirán en las decisiones de los individuos. Por tanto, prestar atención a estos aspectos es necesario desde el punto de vista de la gestión.

Siguiendo el trabajo de Gaudine y Thorne, Connelly et al. en el año 2004 se preguntan por el tipo de emociones que influyen en las tomas de decisiones éticas. Para ello se centran en la elaboración de juicios, segunda etapa del modelo cognitivista de Rest, dado que es una de las etapas menos estudiadas empíricamente. Su estudio ofrece un correlato empírico de las relaciones entre las emociones y las elecciones éticas (2004: 259) mostrando que las emociones tienen una mayor fuerza de relación con las elecciones éticas directamente interpersonales que aquellas que son directamente organizacionales. Y además muestran que hay dos dimensiones a considerar, como ya señalaran Gaudine y Thorne en la emoción que influyen de modo distinto en las tomas de decisiones cognitivas: el estímulo y el sentimiento.

\subsection{Modelo neurocognitivo de toma de decisiones éticas de Reynolds}

En el año 2006 dando un paso más en la utilización de los avances neurocientíficos y neuroéticos para repensar los procesos de toma de decisiones éticas en las organizaciones Reynolds formula el modelo neurocognitivo de toma de decisiones éticas. Este modelo lo sustenta en los estudios de la neuroanatomía, la neurofisiología y la neuroquímica tratando de identificar cómo el cerebro «literalmente»: procesa, interpreta y actúa sobre los estímulos (Reynolds, 2006).

\footnotetext{
4 Para un estudio de las implicaciones de la neurociencia para la antropología y la realidad personal en esta misma línea que recoge la determinación mutua e interdependencia del cerebro racional y emocional SÁnchez Orantos (2015) y Conill (2015)
} 
Bajo este modelo se afirma que el proceso de toma de decisiones éticas implica la existencia y funcionamiento de dos sistemas o ciclos interconectados. El sistema-X y el sistema-Y. El primero es aquel que responde como procesos automáticos, de aprendizaje implícito o incluso intuición a situaciones del día a día y que lo hace de un modo reflexivo pero no consciente frente a la presencia del estímulo pero donde no son activados los pensamientos deliberativos. Aunque sí que existe una reflexión no consciente en tanto en cuanto la situación se contrasta con los prototipos éticos que «(...) son constructos dinámicos que almacenan información descriptiva, evaluativa y prescriptiva en una configuración de signos en red neurales» (Reynolds, 2006: 739). Por tanto lo que afirma Reynolds es que un prototipo puede contener no sólo características del mundo natural, sino también puede contener evaluaciones normativas y recomendaciones prescriptivas (Bowie, 2009: 638).

Es un proceso cíclico iterativo en el que una búsqueda de información o estímulo inicia la estructuración. Pero si el estímulo es demasiado escaso o ambiguo para el objetivo comparativo o si el patrón completo no es reconocible la búsqueda y la reestructuración continua hasta que el sistema puede determinar a cual, si hay alguno, de los prototipos conocidos combina la situación. Todo este proceso se produce de un modo no reflexivo racionalmente. De modo que si este fuera el único sistema de toma de decisiones, como señalan Bowie o Cortina, a la filosofía moral no le quedaría ninguna tarea por cumplir, pero sin embargo no es esta la conclusión que hay que extraer.

Así pues, cabe resaltar, a mi juicio, que esta parte del sistema de toma de decisiones se adecua a la vigencia de las actuaciones o respuestas morales pero no a su validez. Pues tal y como muestra la ética discursiva somos capaces de acometer en nuestra relación con el mundo de la corrección de las normas una distinción entre vigencia y validez de la que no da cuenta el sistema-X (Habermas, 1998; 2000). Por tanto, cabría diferenciar aquí tal y como se viene reclamando desde una ética empresarial crítica (García-Marzá, 2004) la distinción entre moral y ética, o lo que es lo mismo entre respuestas adaptadas a valores, normas y principios vigentes, pero no por ello éticos, y aquellos que encuentran su justificación por lo que todos podríamos querer de modo intersubjetivo ${ }^{5}$. Este proceso es el que se produciría según Reynolds en el C-System. Veamos cómo funciona.

5 Es importante subrayar que mucha de la literatura que trata el tema de la neuroética y su aplicación a las organizaciones hacen una mención explícita a que no van a realizar una distinción entre moral y ética. Un ejemplo lo encontramos en SALVAdor y FolgER (2009:2). A mi modo de ver, aquí radica uno de los mayores errores puesto que tal distinción es fundamental para que se puedan extraer las implicaciones adecuadas a los avances de la neurociencia y su aplicación a la dimensión moral de las diferentes praxis, a saber, derecho, empresa, marketing, economía, educación, etcétera. En este trabajo siguiendo la tradición de la ética discursiva de J. Habermas en su versión ampliada de la razón cordial propuesta por A. Cortina entendemos por «moral» las exigencias que en la vida cotidiana nos exigimos unos a otros en relación y que nos obligan a tratarnos como fines últimos y no como meros medios o instrumentos. 
Si la situación dada no casa con ningún prototipo entonces se requiere de ayuda externa y por tanto comienza la «consciencia», el C-System, o lo que Reynolds denomina el ciclo de razonamiento consciente de orden superior.

El C-System funciona con una lógica simbólica. Y hay dos rasgos fundamentales en su funcionamiento. En primer lugar, este sistema es capaz de realizar un análisis basado en reglas. Bien sean utilitaristas o formalistas o de cualquier otro tipo. Y, en segundo lugar, este sistema realiza un papel regulador del sistema-X. En la actualidad, Reynolds afirma que hay evidencia de que lo hace de dos modos. En primer lugar el sistema-X es dependiente del Sistema-C para su suministro de prototipos, cuando estos los tiene ya reconocidos ya no le hace tanta falta pero dado que el entorno es cambiante, depende de él. En segundo lugar, cada vez se van encontrando más evidencias de que el cingular anterior, actúa a modo de alarma que anima o permite que el sistema-X exprese su incapacidad para combinar de modo adecuado estímulos particulares con algún prototipo conocido y la necesidad del Sistema-C para comprometerle en un proceso activo.

En términos de toma de decisiones, los comportamientos del sistema-C se conceptualizan mejor como un ciclo o bien de racionalización - justificación del juicio emergente - o bien de juicio activo — analizar la situación, aplicar reglas morales y realizar un juicio. De esta última es de la que se ocupa la filosofía moral o ética. En cualquier caso, tanto si el decisor racionaliza o realiza un juicio activo, se produce un proceso de reestructuración (Reynolds, 2006: 741)

Según Reynolds este modelo neurocognitivo de dos ciclos pone en cuestión la linealidad del modelo de toma de decisiones éticas de Rest teniendo en cuenta la base neural del cerebro y su funcionamiento en las tomas éticas de decisión. Los tres aspectos que quedan contrapuestos son los siguientes. En primer lugar, el modelo neurocognitivo muestra que en ocasiones no se llega a producir una toma de conciencia ni un reconocimiento de la cuestión ética, puesto que el X-System genera la respuesta sin ser consciente basándose en decisiones intuitivas no-reflexivas. En segundo lugar, porque el modelo de Rest reconoce el C-System pero para el modelo neurocognitivo este es un paso más pero muchas veces poco común, mientras que para el modelo cognitivista es el núcleo central del mismo. En tercer lugar, el modelo de Rest considera que hay una diferencia entre formular una intuición y la actuación mientras que bajo el modelo neurocognitivo esta distinción es irrelevante. Irrelevancia que explica porque considera que depende de si la situación concreta la persona puede tener la intención de hacer algo y finalmente comportarse de otro modo atendiendo a si la situación es hipotética o real. Así pues dado que las situaciones son distintas los prototipos que se encuentran y se utilizan son distintos.

Por tanto, bajo este modelo se considera que en la investigación de la toma de decisiones éticas en las organizaciones cabe considerar al menos dos aspectos fundamentales. En primer lugar que en el modelo neurocognitivo, a diferencia del cognitivo, no hay linealidad y se debe entender que hay una simultaneidad —en la mayoría de las ocasiones - entre descripción, juicio y prescripción en las decisiones reflexivas. Y, en segundo lugar, que el 
razonamiento tradicional es poco usual, que el más común es el irreflexivo y que cuanto más se utiliza éste más puede trabajar el C-system para adaptarse a los nuevos escenarios. Es decir, en la mayoría de las situaciones se utiliza el $\mathrm{X}$-system — reflexivo- y frente a situaciones nuevas o complicadas el lineal de C-system - racionalista-.

Siguiendo esta propuesta del modelo neurocognitivo para la toma de decisiones éticas se diferencia entre el juicio activo y el juicio reflexivo. El juicio activo es el resultado de un proceso analítico deliberativo y se construye sobre las reglas morales actuales disponibles para elaborar un juicio. Por su parte, el juicio reflexivo es la consecuencia, por el contrario, de la combinación de patrones no conscientes, y los juicios encapsulados en los prototipos podrían estar fundamentados en reglas morales que son diferentes de aquellas que actualmente se utilizan. Esto explicaría, según sus defensores, por ejemplo que un mismo sujeto contestara a los dilemas de Kohlberg o al modelo de Rest y estuviera en un nivel alto y sin embargo al salir a la calle frente a un dilema real, donde echara mano de prototipos anteriores reflexivos pero no deliberativos y podría quedar en el primer nivel del juicio moral.

Ahora bien, a mi juicio, cabe considerar si de lo que están hablando no es simplemente de un proceso de toma de decisiones situado en el nivel de conciencia moral convencional y en el segundo lugar en el nivel de conciencia moral postconvencional crítica, en terminología kohlbergiana. Y que lo único que añade este modelo son los tiempos de respuesta y la localización de las zonas del cerebro más implicadas en estos dos niveles de razonamiento. Por tanto, aunque subraye elementos que han sido poco trabajados como son las emociones y las intuiciones en las organizaciones, a mi modo de ver, no es un nuevo modelo de toma de decisiones éticas. Aspecto que sí cabría tener en consideración y obligaría a un desarrollo de los mismos en los estudios de la ética empresarial. De este aspecto se ocupa el trabajo en el siguiente apartado.

\section{Cuatro caminos de Reconocimiento de la Emoción EN Las organizaciones: BRECHAS AL MITO DE LA RACIONALIDAD}

En el ámbito de las organizaciones y la ética empresarial las emociones han sido infravaloradas a favor de la racionalidad tanto por parte de las propuestas normativas como por los desarrollos prácticos. La razón ha gozado de una atención preeminente. En los inicios del siglo Xxi algunos teóricos del ámbito organizativo como Weick (1995; 2001) o Fineman (1996; 2000) fueron precursores en el reconocimiento de la importancia de las emociones en las organizaciones. Estos autores sin el apoyo que posteriormente ha brindado el avance de la neuroética y la biología evolutiva argumentaban que la distinción racional/emocional es una falsa dicotomía dado que «muchas estrategias organizacionales racionales están sustentadas en fundamentos emocionales y mucho de lo que describimos como racional es de hecho emocional» (Fineman, 
1996: 550) de modo que los procesos organizativos son en sí mismos altamente emocionales.

Los estudios neurocientíficos —especialmente desarrollados por Damasio y LeDoux - han sido vitales para mostrar que durante mucho tiempo ha existido un error en el modo en el que se ha abordado el binomio emoción-cognición, sobre todo porque se ha tratado de estudiar en ocasiones el pensamiento y el comportamiento humano sin contar con las emociones. Es lo que magistralmente ilustró Damasio en su estudio El Error de Descartes (1999). Este autor argumenta que nuestros sistemas cognitivos y emocionales trabajan conjuntamente y el razonamiento depende de lo que él denomina «marcadores somáticos» del cuerpo físico.

El punto de conexión crítico de los sistemas fisiológicos y neurológicos reside en el sistema límbico del cerebro que según los estudios desarrollados por Damasio son los que conducen nuestras respuestas emocionales. Los sistemas emocionales del cerebro comprenden un sistema complejo de rutas neurales -neural pathways - conectadas a un núcleo distribuido a través del cerebro humano. Siguiendo esta línea de trabajo Joseph LeDoux (1999) trabajó sobre todo con la compresión de cómo en la emoción del miedo está implicada la amígdala. Ahora bien, Damasio (2001) también explica que la amígdala no está implicada en todas las emociones. Las emociones positivas así como la emoción negativa del disgusto están procesadas en rutas neurales que no están unidas a la amígdala.

Estos descubrimientos y avances en el conocimiento de las bases neurofisiológicas del cerebro de nuestras emociones implican que nuestras emociones no siempre están bajo control. Los circuitos neurales que están influidos por la amígdala controlan nuestras respuestas cardiovasculares, respiratorias, automáticas, faciales y endocrinas. El punto relevante aquí es que estas respuestas se inician preconscientemente y tienen y poseen unos resultados a los que sí que podemos acceder en nuestra intimidad consciente y son visibles externamente.

Y en el ámbito de la moralidad, el estudio de las bases neurales ha permitido llegar a conocer que algunas partes y regiones del cerebro juegan un papel relevante en los procesos afectivos y emocionales — cortex orbitofrontal medial, la amígdala, y el cingulate anterior- puesto que éstas se activan en individuos que están expuestos a estímulos relevantes moralmente mucho más que en individuos que no son expuestos a estas situaciones. El trabajo empírico desarrollado demuestra que las emociones son fundamentales no sólo para tomar conciencia moral - exaltación y sentimiento de la emoción- sino también en la formulación de los juicios morales, así como que son relevantes en los procesos de toma de decisiones éticas. Estos estudios también han permitido mostrar la existencia de mecanismos neurales unidos al juicio moral con emociones particulares, por ejemplo los juicios sobre situaciones de justicia han sido asociados con la emoción de la satisfacción (Salvador y Folger, 2009).

A la luz de estos conocimientos sobre las bases biológicas y neurológicas de las emociones comienzan a surgir enfoques tanto desde perspectivas filosóficas, 
organizativas o neurobiológicas que retan a los modelos organizativos cognitivistas y racionalistas mayoritarios en toda la literatura tanto de teoría organizativa como de la ética empresarial. Llegando a hablarse del «mito de la racionalidad» porque consideran que esta de algún modo está relacionada con la emoción y que no ha sido considerada en tales modelos racionalistas.

Dougherty y Drumheller ponen el acento en la brecha que se ha producido en el terreno de las organizaciones respecto a este mito. Brecha que se ha generado en las teorías organizacionales por la confluencia de diferentes propuestas que no le dan el mismo valor ni posición a las emociones, pero que han conseguido que se replanteara el mito de la racionalidad en las organizaciones. Las estrategias utilizadas las agrupan bajo cuatro caminos distintos que se analizan a continuación (Dougherty y Drumheller, 2006: 218-219) tratando en este estudio de relacionarlas con las posiciones que desde la filosofía moral las sustentarían.

El primero es el que afirma que la racionalidad no es sólo razón puesto que las emociones pueden nublar el juicio, por tanto, afirman quienes utilizan esta senda que hay que reconocerlas aunque luego apuestan por la vía de segmentar cuidadosamente las expresiones emocionales y apuntan que preferiblemente deberían ser extirpadas de la organización. Su fundamento filosófico normativo lo encontraríamos en las corrientes del estoicismo que realiza un reconocimiento explícito de las mismas para luego dejarlas a un lado, pero también las corrientes contemporáneas kantianas y utilitaristas en su justificación de la racionalidad práctica parecen optar por estrategias muy cercanas a este camino.

El segundo camino advierte que las emociones pueden dominar la racionalidad por lo que el objetivo es conseguir que las emociones se pongan al servicio y estén dominadas por la racionalidad. En este caso hay un reconocimiento del papel de la emoción y se pretende integrar en la gestión pero en un papel de subordinación y manteniendo la separación tajante entre la emoción y la razón. Esta posición ha sido analizada y argumentada filosóficamente por Victoria Camps (2011) de la mano de Aristóteles, Spinoza y Hume, sosteniendo que las emociones son disposiciones mentales que generan actitudes, porque, argumenta, si bien las emociones tienen una base fisiológica no se reducen a ella porque puede el sujeto llegar a dominarlas. Considera Camps en este sentido que la ética en su quehacer no puede prescindir de la emoción «(...) porque una de sus tareas es, precisamente, poner orden, organizar y dotar de sentido a los afectos o las emociones»(Camps, 2011: 25).

Por tanto, la ética empresarial tendrá también que considerarla para poder guiarlas o gobernarlas en un sentido u otro en la dirección y gestión de empresas. Desde esta perspectiva las emociones no sólo se padecen o se sufren sino que son actitudes mentales que generan actitudes en un sentido o en otro. Entre otras razones porque se sigue la posición racionalista y emotiva de Spinoza desde la que la personalidad humana es un continuo en el que los afectos y la razón se complementan (Camps, 2011: 66). Donde es necesario conocer los afectos para poder gobernarlos y conducirlos, puesto que sólo son perjudiciales cuando provienen de ideas inadecuadas. De ahí que el objeto de la Ética 
de Spinoza sea transformar las ideas inadecuadas que proceden del exterior en ideas adecuadas que buscan la esencia humana, que brotan de la razón y apuntan a propiedades comunes de la cosas, como ley de conatus o esfuerzo, por la que el deseo es vivir y vivir bien (Camps, 2011: 69ss). De este modo, si la esencia humana está en el deseo, es necesario que continuamente se nos presente lo justo como deseable (Camps, 2011: 79). De ahí la importancia de educar el deseo y orientarlo, así como de dotar al ser humano de un método para ordenar la vida.

En tercer lugar, se presenta el enfoque que considera que razón y emoción están entrelazados de tal modo que no se pueden separar porque la racionalidad es aplastantemente emocional. En esta posición se podría situar la postura normativa neoestoica de Martha Nussbaum (2008) que al definir la emoción como parte esencial del razonamiento ético, no sólo como motivaciones que apoyan o socaban nuestra elección sino porque en sí mimas las emociones están imbuidas de inteligencia y de discernimiento. Y por tanto, afirma la autora, no pueden dejarse de lado al dar cuenta del juicio moral o de la acción moral. Razón y emoción están interrelacionadas porque las emociones son juicios cognitivos-evaluativos y eudaimonistas. Por tanto, las emociones tienen que ver con la vida humana plena que el sujeto posee, el pensamiento está inserto ya en la emoción (Nussbaum, 2008: 52). Así pues las emociones son juicios evaluativos cognitivos que están basados en creencias y enjuician el mundo desde el esquema de los proyectos y objetivos personales. Así pues, aunque la base biológica de las emociones sea común éstas sufren gran variación porque las creencias pueden ser, de hecho lo son, modeladas por las normas sociales y por la propia historia individual. La variación social posee diferentes fuentes y niveles que da lugar a que las sociedades vayan transmitiendo concepciones diferentes acerca de los objetos apropiados para una emoción, las cuales, una vez más, moldean la experiencia, así como el comportamiento (Nussbaum, 2008: 180-194).

Y, en cuarto lugar, basado en aquellos avances neurocientíficos en los que se le concede a la emoción un privilegio sobre la razón, basado en la intuición moral que precede al juicio moral. Esta posición ha sido especialmente defendida por la teoría del Intuicionismo social defendido por el psicólogo Jonathan Haidt. Desde esta teoría la intuición moral se entiende como una respuesta rápida (automática), no-deliberada (no-inferencial) de experiencias evaluativas que a menudo están cargadas emocionalmente (Haidt, 2012 y Weaver et al. 2014). En la literatura referente a la «intuición moral» existen diferentes esquemas desde los que abordarla, pero destacan: a) la intuición moral se considera como un proceso no-deliberativo fundamentalmente de formación de juicios rápidos que es rápida y rutinizada. Donde se estudia como un aprendizaje implícito; y b) la intuición moral es innata y existen invariables intuiciones morales, al estilo de capacidades innatas morales. Estas se configuran como módulos que se desarrollan dentro de un «marco evolutivo». Ahora bien, lo que mantienen desde esta estrategia mina el «mito de la racionalidad» dado que 
sostienen que la emoción tiene un privilegio sobre la razón tal y como antes veíamos en el modelo neurocognitvo de toma de decisiones ético de Reynolds.

Una vez hemos visto los modos diferentes en los que se ha ido recuperando la emoción en el terreno de las organizaciones apoyado por esa recuperación también filosófica de la misma se puede afirmar que la emoción ha irrumpido con mayor fuerza en los modelos organizativos sobre todo de la mano de la neurociencia y a mi modo de ver, debería estudiarse con detenimiento qué implicaciones tiene el avance de la neuroética de la ciencia en el desarrollo presente de la ética empresarial.

\section{IMPLICACIONES PARA LA ÉTICA EMPRESARIAL Y SU DESARROLLO}

Los avances de la neuroética de la ciencia representan serios retos e implicaciones para la orientación y desarrollo actual de la ética empresarial. El diálogo y el trabajo interdisciplinar se hace necesario especialmente porque la ética empresarial crítica tiene la pretensión, como se ha señalado, de orientar las tomas de decisiones sobre la cultura, la política y la estrategia organizativa y tiene unas repercusiones prácticas en sentido de prudencia, justicia y responsabilidad muy elevadas. Por tanto, cabe tomar buena nota de los retos que supone ese diálogo entre ética empresarial, neurociencia organizacional y neuroética para su desarrollo.

En primer lugar, la consideración de un sistema de toma de decisiones ético que es cognitivo y afectivo al mismo tiempo conduce al desarrollo de modelos teórico-prácticos que reconozcan tanto los afectos (emociones y sentimientos) como la razón (interés, normas, valores). Ahora bien, como señala Bowie «Mostrar como funcionan los procesos de toma de decisiones no es lo mismo que justificar una decisión» (2009: 637) por lo que el desarrollo de toma de decisiones deberá contemplar esta complejidad entre emociones, intuiciones y razones pero con ello no se está justificación moralmente las decisiones. De esta tarea deberán seguir ocupándose los desarrollos normativo-filosóficos.

En segundo lugar, trabajar en el desarrollo de buenas prácticas que se vayan estableciendo como prototipos sobre los que formar en las organizaciones puesto que como evidencia el estudio neuroético en las tomas de decisiones éticas las emociones o quizás los procesos no-reflexivos e inconscientes que encajan con prototipos juegan un papel más dominante en el día a día que las reglas éticas o la reflexividad ética. Por tanto, desde la ética empresarial deben desarrollarse guías de buenas prácticas aplicadas a cada uno de los ámbitos y sectores organizativos.

En tercer lugar, trabajar sobre las intuiciones morales que se poseen con respecto a las relaciones entre los diferentes stakeholders de modo que se expliciten y puedan ser analizadas, reforzadas o confrontadas, de modo que sea la organización quien tome la dirección de los afectos y no al contrario. Por tanto que se produzca un gobierno de las emociones desde el reconocimiento 
de las intuiciones. En este sentido tanto los desarrollos empíricos como las propuestas normativas deben trabajar codo con codo en los terrenos de la ética empresarial.

En cuarto lugar, al estudiar la gestión de las relaciones con los diferentes stakeholders de la empresa, es decir aquellos que son afectados por el logro de los objetivos de la organización (Freeman, 1984; Freeman et al., 2010; González Esteban, 2007) cabe ahora hacer hincapié no sólo en la explicitación y discusión sobre las razones de sus intereses sino también la explicitación de las intuiciones que se mueven de fondo en las relaciones así como en las emociones. Esta tarea sin duda alguna requiere del diseño de procesos organizativos que favorezcan el diálogo basado en el reconocimiento recíproco en la organización.

En quinto lugar, tiene una gran implicación en el contenido y el modo en el que se realiza la formación en ética empresarial (Reynolds, 2006), tanto en las universidades y escuelas de negocio como en las organizaciones. Deberá ser de corte más dialógico de modo que prime la educación y no la instrucción. El modelo neurocognitivo indica que la educación ética es posible, pero que en la enseñanza se deberá tener en cuenta sobre qué ciclo se está trabajando para ser efectivos. Para ser efectivos la educación ética debería centrarse en las estructuras mentales de los prototipos y las reglas morales, así como también en el razonamiento consciente de orden superior que debería ser deliberativo. Una de las principales funciones del ciclo de razonamiento consciente de orden superior es el de reestructurar y refinar los prototipos, de modo que estos reflejen de modo más apropiado los prototipos compartidos de la unidades socioculturales relevantes.

En sexto lugar, tiene implicaciones en la cultura organizativa (Reynolds, 2006) tanto en el reconocimiento de la propia cultura corporativa como sobre los mecanismos para modificarla. La cultura organizativa está formada por estructuras formales e informales. En cuanto a las estructuras formales encontramos la política y estrategia de la organización que se refleja por ejemplo en los sistemas de compensación y sistemas de valoración de la productividad. Por su parte, los mecanismos informales se pueden ver reflejados en el comportamiento de los líderes o las presiones externas. En ocasiones las empresas poseen culturas corporativas que chocan con los valores, normas y principios compartidos y en otras los siguen sin ser capaces de situarse en posiciones críticas con respecto a los que la sociedad acepta como válidos cuando en realidad sólo están vigentes. En este sentido el modelo neurocognitivo propuesto por Reynolds apunta que esto podría producirse por cuatro razones. En primer lugar, porque los prototipos compartidos a través de la organización están mal etiquetados o identificados, por lo que se produce una falta de sintonía con el entorno. En segundo lugar, porque estos prototipos asignan valores normativos incorrectos a acciones particulares. En tercer lugar, porque la cultura organizativa enfatiza una regla moral que es inapropiada o genera prescripciones vagas. $\mathrm{O}$, en cuarto lugar, porque las reglas morales de la organización no son aplicadas de modo apropiado. En cualquier caso, cualquiera de estas condiciones pueden 
conducir a comportamientos no éticos. Así pues los mecanismos para cambiar la cultura organizativa en una dirección más ética reside en comprender los prototipos que están siendo compartidos por la organización así como sus reglas morales porque estos son los que están siendo reforzados culturalmente de modo formal o informal. Una vez descubiertos cabe trabajar de nuevo con ellos para cambiarlos por otros que den como resultado un comportamiento ético.

\section{CONCLUSIÓN: CAUTELA Y NECESIDAD DE UN TRABAJO INTERDISCIPLINAR}

Sin duda alguna el avance de la neuroética de la ciencia así como su aplicación al ámbito organizativo en la neurociencia organizacional ha supuesto un mejor conocimiento de las bases fisiológicas y el funcionamiento de las relaciones que se establecen en las empresas. Este conocimiento permite descubrir como se ha visto a lo largo de este trabajo que las emociones tienen que ser reconocidas e incorporadas en el desarrollo normativo y práctico de la ética empresarial. Así pues, las emociones y las razones deben ser tratadas de modo entrelazado, tanto en el diseño de las tomas de decisiones, de los procesos formativos, de las relaciones con los stakeholders así como en la configuración de la cultura organizativa y de los liderazgos.

Ahora bien, es fundamental que dado el modo en el que se desarrollan muchas de las propuestas y estudios desde el ámbito neurocientífico no perdamos de vista algunas cautelas que cada vez se tienen más en cuenta, pero que no debemos por el momento dejar de subrayar. En primer lugar, no se puede olvidar que la neurociencia organizacional y la neuroética utilizan herramientas de investigación empírica que describe «lo que hay» y además lo hace sin poder establecer de modo definitivo que una región concreta del cerebro sea necesaria para un proceso cognitivo específico. Por tanto, se necesita que los datos recabados por estas técnicas experimentales sean interpretados y en ocasiones tales interpretaciones pueden ser erróneas, bien porque se infieren conclusiones sobre estudios de laboratorio que no se corresponden con las situaciones reales del día a día, bien porque las muestras que se toman son muy reducidas. En segundo lugar, cabe tener cautela en la exposición y divulgación de los resultados, pues se ha demostrado que en diversos estudios se corre el riesgo de que pequeños avances en el conocimiento derivados de estudios experimentales al ser mostrados con las imágenes cerebrales producen el efecto de certeza y fiabilidad cuando en realidad sólo muestran la actividad de una parte del cerebro, pero que muchas gentes infieren que estas «muestran la realidad de la moralidad» o descubrir las «causas primeras del comportamiento» confiriéndole un poder a las técnicas neurocientíficas que no poseen. (Pallarés Dominguez, 2011; Pallarés Dominguez y González Esteban, 2016). En tercer lugar, se debe pedir cautela porque estos estudios se realizan en el laboratorio donde la interactuación entre diferentes actores se ve constreñido por situaciones muy dirigidas y poco espontáneas en las relaciones que además se refieren a situaciones 
dilemáticas de carácter binario y no problemáticas, por lo que las conclusiones que de ellos se extraen poco nos dicen de las situaciones que se producen en las interacciones de las personas en grupos como es la práctica empresarial. Por tanto, se debe pedir cautela pero también un trabajo interdisciplinar con la filosofía moral y las distintas éticas aplicadas.

La neuroética de la ciencia ha avanzado mucho en el conocimiento del funcionamiento del cerebro y también nos ha hecho percatarnos de la importancia de la emoción y la intuición en la vida moral pero dicho avance debe ir unido a la pregunta por el fundamento filosófico de la obligación moral, aspecto que debe hacer junto con y en diálogo con las teorías éticas y las éticas aplicadas. Por tanto, cabe seguir avanzando porque la fuerza normativa que posee hoy por hoy la neurociencia por sí sola está entre dicho (Salles, 2013; Bowie, 2009) entre otras razones porque como afirma con una gran claridad Cortina una cosa es la base y otra el fundamento de la moralidad. Es decir, el peligro de tomar una explicación, que es de lo que nos habla la neurociencia, sobre las bases fisiológicas del cerebro necesarias para la vida moral en las organizaciones y otra bien distinta considerar que pretenden darnos el fundamento de la moralidad (Cortina, 2011: 46-47; 2012b; 2013:129). A mi juicio para que la neurociencia organizacional pueda ofrecer más orientaciones a la ética empresarial tiene que trabajar de modo interdisciplinar con ella y ser una auténtica neuroética aplicada a la empresa. Tarea que resta por acometer en un futuro.

\section{BibliografíA}

Becker, W. J. y Cropanzano, R., «Organizational neuroscience: The promise and prospects of an emerging discipline», Journal of Organizational Behavior, núm.31, 2010, pp. 1055-1059.

Becker, W. J., Cropanzano, R. y Sanfey, A. G., «Organizational Neuroscience: Taking Organizational Theory Inside the Neural Black Box», Journal of Management, núm. 4, vol. 37, 2011, pp. 933-961.

BowIE, N. E., «How Emprirical Research in Human Cognition Does and Does not Affect Philosophical Ethics», Journal of Busines Ethics, núm. 88, 2009, pp. 635-643.

Butler, M. J.r. y SEnior, C., «Toward an Organizational Cognitive Neuroscience», Annals of the New York Academy of Sciences, 1118, 2007, pp. 1-17.

Calvo, P. y González Esteban, E., «Neuroeconomía ¿un saber práctico?», en: GarcíaMarzá, D. y Feenstra, R. (ed.), Ética y neurociencias. La aportación a la política, la economía y la educación, Servei de Publicacions Universitat Jaume I, Castelló, 2013, pp. 93-116.

CAmps, V., El gobierno de las emociones. Herder, Barcelona, 2011.

Conill, J., «La realidad personal en perspectiva neurocientífica. La aportación zubiriana», Pensamiento, núm. 266, 2015, pp. 253-271.

Conill, J., «Neuroeconomía y neuromarketing. ¿Más allá de la racionalidad maximizadora?, en: Cortina, A. (ed.), Neurofilosofía práctica, Comares, Granada, 2012, pp. 30-64. 
Connelly, S., Helton-Fauth, W. y Mumford, M. D., «A Managerial In-Basket Study of the Impact of Trait Emotions on Ethical Choice», Journal of Business Ethics, núm 51, 2004, pp. 245-267.

Cortina, A., Conill, J., Domingo, A. y García-Marzá, D., Ética de la empresa. Hacia una nueva cultura empresarial, Trotta, Madrid, 1994.

Cortina, A., «Ética del discurso: ¿un marco filosófico para la neuroética?, Isegoría. Revista de Filosofía Moral y Política, núm.48, 2013, pp. 127-148.

Cortina, A., «La responsabilidad social corporativa y la ética empresarial» en: Galán, J.I. y SÁEnz de Miera, A. (eds.), Reflexiones sobre la Responsabilidad Social Corporativa en el siglo XXI, Ediciones Universidad de Salamanca, Salamanca, 2012a, pp. 69-88.

Cortina, A., "Neuroética: presente y futuro», en: Cortina, A. (ed.), Guía Comares de Neurofilosofía práctica, Comares, Granada, 2012b, pp. 9-38.

Cortina, A., Neuroética y neuropolítica, Tecnos, Madrid, 2011.

Cropanzano, R. y Becker, W. J., «The Promise and Peril of Organizational Neuroscience: Today and Tomorrow», Journal of Management Inquiry, núm.3, vol. 22, 2013, pp. 306-310.

Damasio, A., El error de Descartes, Crítica, Barcelona, 1996.

Damasio, A., La sensación de lo que ocurre. Cuerpo y emoción en la construcción de la conciencia, Debate, Barcelona, 2001.

Damasio, A., Y el cerebro creó al hombre: ¿cómo pudo el cerebro generar emociones, sentimientos, ideas y el yo?, Destino, Barcelona, 2010.

Dougherty, D. S. y Drumheller, K., «Sensemaking and Emotions in Organizations: Accounting for Emotions in a Rational(ized) Context», Communication Studies, núm.2, vol.57, 2006, pp. 215-238.

Evers, K., Neuroética. Cuando la materia se despierta, Katz, Madrid, 2009.

Feenstra, R., «La irrupción de la neuropublicidad y sus debates éticos», Daimón Revista Internacional de Filosofía, núm. 59, 2013, pp. 45-56.

Fineman, S., «Emotion and organizing», in ClegG, S. R., Hardy, C. y Nord, W. R. (Eds.), Handbook of organization studies, London, Sage, 1996, pp. 543-564.

Fineman, S. (ed.), Emotion in organizations, Newbury Park, Sage, 2000.

FLACK, J. C. y DE WAAL, F. B. M., «Monkey business and business ethics: evolutionary origins of human morality», Business, Science, and Ethics, 2004, pp. 7-41.

Folger, R. y Salvador, R., «Is Management Theory Too «Self-ish»?», Journal of Management, núm. 6, vol. 34, 2008, pp. 1127-1151.

Freeman, R. E., Strategic management. A Stakeholder Approach, Pitman, Toronto, 1984.

Freeman, R.E., Harrison, J. S., Wicks, A. C., Parmar, B. L., y Colle, S., Stakeholder Theory. The State of the Art, Cambridge University Press, Cambridge, 2010.

García-Marź, D., Ética empresarial. Del diálogo a la confianza. Tecnos, Madrid, 2004.

Gaudine, A. y Thorne, L., "Emotion and Ethical Decision-Making in Organizations», Journal of Business Ethics, núm. 31, 2001, pp. 175-187.

GonzÁLEZ EsteBAn, E., «La teoría de los stakeholders: un puente para el desarrollo práctico de la ética empresarial y de la responsabilidad social corporativa», Veritas. Revista de Filosofía y Teología, vol. II, no 17, 2007, pp. 205-224.

Habermas, J., Facticidad y validez. Sobre el derecho y el Estado democrático de derecho en términos de teoría del discurso, Trotta, Madrid, 1998

Habermas, J., Aclaraciones a la Ética del discurso, Trotta, Madrid, 2000.

HAIDT, J., «El perro emocional y su cola racional: un enfoque intuicionista social del juicio moral» en Cortina, A. (ed.), Guía Comares de Neurofilosofía Práctica, Comares, Granada, 2012, pp. 159-216. 
JonEs, T., «Ethical Decision-Making by Individuals in Organization: An Issue-contingent Model», Academy of Management Review, núm. 16, vol. 2, 1991, pp. 366-395.

Kohlberg, L., Essays on Moral Development, vol. I, San Francisco, 1981.

LeDoux, J., El cerebro emocional, Ariel, Barcelona, 1999.

Lindebaum, D. y Zundel, M., «Not quite a revolution: Scrutinizing organizational neuroscience in leadership studies», Human Relations, núm. 6, vol. 66, 2013, pp. 857-877.

Lozano Aguilar, J. F., «Toma de decisión ética en la gestión de empresas: la aportación de la neuroética», Daimón Revista Internacional de Filosofía, núm. 59, 2013, pp. 183196.

Morgado, I., Cómo percibimos el mundo: una exploración de la mente y los sentidos, Ariel, Barcelona, 2012.

Nussbaum, M. C., Paisajes del pensamiento. La inteligencia de las emociones, Paidós, Barcelona, 2008.

Pallarés Dominguez, D., "Críticas y orientaciones para el estudio en neuroética», Recerca. Revista de Pensament i Análisi, núm. 13, 2013, pp. 85-102.

Pallarés Dominguez, D. y González Esteban, E. «The Ethical Implications of Considering Neurolaw as a New Power», Ethics \& Behavior, vol. 26, núm. 3, 2016, pp. 252-266.

Rest, J. R., Development in judging moral issues, MN Univesrity of Minnesota Press, Minneapolis, 1979.

Rest, J. R., Moral development: Advances in research and theory, Praeger, New York, 1986.

REYNolds, S. J., "A Neurocognitive Model of the Ethical Eecisión-making Process: Implications for study and practice», Journal of Applied Phsicology, núm. 4, vol.91, 2006, pp. 737-748.

SAlLES, A., "On the normative implications of social neuroscience», Recerca. Revista de Pensament i Análisi, núm. 13, 2013, pp. 29-42.

Salvador, R. y Folger, F., "Business Ethics and the Brain», Business Ethics Quarterly, núm. 1, vol. 19, 2009, pp. 1-31.

SÁnchez ORAntos, A., «Retos que plantean las neurociencias a la antropología filosófica», Pensamiento, núm. 269, 2015, pp. 1295-1308.

WEICK, K. E., Sensemaking in organizations, Thousand Oaks, Sage, 1995.

WEICK, K. E., Making sense of the organization, Malden, Backwell, 2001.

Weaver, G. R., Reynolds, S. J. y Brown., M. E., «Moral Intuition: Connecting Current Knowledge to Future Organizational Research and Practice», Journal of Management, núm.1, vol. 40, 2014, pp. 100-129.

Universitat Jaume I,

Avda. Sos Baynat, s/n, E-12071 Castellón

Elsa GonZÁlez Esteban

Elsa.Gonzalez@uji.es

[Artículo aprobado para publicación en octubre de 2016]. 